\title{
Lyman- $\alpha$ emission in the Martian proton aurora: Line profile and role of horizontal induced magnetic field
}

\author{
J.C. Gérard ${ }^{\mathrm{a}, *}$, B. Hubert ${ }^{\mathrm{a}}$, B. Ritter ${ }^{\mathrm{a}, \mathrm{b}}$, V.I. Shematovich ${ }^{\mathrm{c}}$, D.V. Bisikalo ${ }^{\mathrm{c}}$

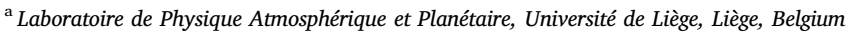 \\ ${ }^{\mathrm{b}}$ Observatoire Royal de Belgique, Brussels, Belgium \\ ${ }^{\mathrm{c}}$ Institute of Astronomy of the Russian Academy of Sciences, Moscow, Russia
}

\section{A B S T R A C T}

Enhancements of the dayside Lyman- $\alpha$ emission by as much as $\sim 50 \%$ have been observed between 120 and $130 \mathrm{~km}$ in the lower Martian thermosphere from the Mars Express and MAVEN satellites, usually following solar events such as coronal mass ejections and corotating interaction regions. They have been assumed to be optical signatures of proton aurora related to an increase in the solar wind proton flux hitting Mars' bow shock. We present model simulations of the Lyman- $\alpha$ line profiles at different altitudes. These are partly guided by in situ measurements of the energy spectrum of protons in the magnetosheath region by the SWIA instrument on board the MAVEN spacecraft. We show that the auroral Lyman- $\alpha$ line profile is significantly broader than the hydrogen core of the planetary thermal $\mathrm{H}$ atom. Consequently, most of the auroral emission is produced outside the optically thick hydrogen core and creates the observed intensity enhancement. Simulations with incident energetic hydrogen atoms (H ENAs) produce a somewhat broader line profile. Monte Carlo calculations are made separately for incident solar wind protons and $\mathrm{H}$ ENAs produced by charge exchange in the hydrogen corona. Absorption by $\mathrm{CO}_{2}$ along the line of sight significantly affects the intensity distribution in the lower thermosphere. The calculated altitude of the peak emission for both types of incident particles is consistent with the observed characteristics of the proton aurora. We show that the presence of a horizontal induced magnetic field somewhat increases the line width and decreases the altitude of the emission peak as a consequence of the magnetic barrier effect on proton precipitation. The brightness of the Lyman- $\alpha$ emission also drops as a result of increased magnetic shielding of the protons.

\section{Introduction}

Three different types of aurora have been discovered on Mars through their ultraviolet emission signatures. The first one, the discrete aurora, was initially detected by Bertaux et al. (2005) with the Imaging Ultraviolet Spectrograph (SPICAM) on board Mars Express. Subsequent studies (Leblanc et al., 2006; Gérard et al., 2015) indicated the close relationship between the location of these nightside detections and the cusp-like topology of the crustal magnetic field. A second type, the diffuse aurora, was observed with the Imaging UV Spectrograph (IUVS) on board MAVEN as the spectrograph was scanning the nightside planet's limb over a wide range of latitudes and longitudes (Schneider et al., 2015). They occur following solar activity events such as coronal mass ejections or compression events and were observed in the northern hemisphere in region, although they are believed to be global. Finally, enhancements of the Lyman- $\alpha$ emission have been occasionally seen in the dayside thermosphere with SPICAM (Ritter et al., 2018) and IUVS (Deighan et al., 2018) instruments. The peak altitude of the additional emission ranged from about 120 to $140 \mathrm{~km}$ and the excess brightness was between a few hundred Rayleighs (R) and 3 kilo Rayleighs (kR). The lowest intensity values approximately correspond to the detection limit of the spectrographs in the presence of background resonance scattering dayglow emission but weaker events likely occurred and remained undetected. The SPICAM events correspond to the declining phase of solar cycle 23 while the IUVS detections were made following the (moderately active) peak of cycle 24 . They show similar shapes of the Lyman- $\alpha$ limb profile enhancement and comparable peak intensities. These detections were associated with enhanced solar activity conditions.

Hydrogen ENAs result from the interaction of solar wind protons with the Martian neutral hydrogen exosphere upstream of the bow shock and retain their characteristic energy $\sim 1 \mathrm{keV}$ in the solar wind. Deighan et al. (2018) observed a correlation between the presence of auroral Lyman- $\alpha$ and enhancements of the penetrating proton flux measured with the Solar Wind Ion Analyzer (SWIA) instrument on board MAVEN. These penetrating protons are the product of charge exchange of penetrating ENAs colliding with the constituents of the Martian thermosphere (Kallio et al., 1997; Kallio and Barabash, 2001; Bisikalo et al., 2018). They lose their energy by ionizing, dissociating and exciting the atmospheric constitutents (mostly $\mathrm{CO}_{2}, \mathrm{O}, \mathrm{CO}$ and $\mathrm{N}_{2}$ ), heating the ambient gas and radiating. Once inside the atmosphere, a balance is established in the beam between charge exchange and

\footnotetext{
* Corresponding author.

E-mail address: jc.gerard@ulg.ac.be (J.C. Gérard).
} 
electron stripping, leading to an equilibrium fraction of protons and $\mathrm{H}$ ENAs.

Monte Carlo simulations of the interaction of auroral electrons with the Martian atmosphere have been presented by Soret et al. (2016) for the discrete aurora and by Gérard et al. (2017) for the higher energy electron precipitation in the diffuse aurora. They calculated the production rate of the main ultraviolet auroral emissions for different electron characteristic energies. Bisikalo et al. (2017) described Monte Carlo simulations indicating that magnetic mirroring of electrons in the presence of a crustal magnetic field leads to an intensification of the backscattered electron flux and a reduction of the total auroral emitted power. Shematovich et al. (2011) presented Monte Carlo simulations of the interaction of an energetic $\mathrm{H}^{+}-\mathrm{H}$ precipitation with the atmosphere based on proton fluxes measured by ASPERA 3 on board Mars Express. They analyzed the effects of the presence of a horizontal induced magnetic field. They found that $12 \%$ of the energy flux carried by the precipitating protons is backscattered by the Martian upper atmosphere in the absence of a magnetic field. This fraction increased up to $92 \%$ in the presence of an induced magnetic field of $50 \mathrm{nT}$. They concluded that the induced magnetic field plays a crucial role in the transport of energetic $\mathrm{H}^{+}-\mathrm{H}$ in the Martian upper atmosphere. Measurements based on the Mars Global Surveyor magnetometer and the ASPERA-3 MARSIS low-frequency echoes indicate that the intensity of the draped B-field varies significantly. It was shown to depend on solar zenith angle (Brain et al., 2003; Akalin et al., 2010), statistically dropping from $\sim 40 \mathrm{nT}$ near the subsolar point to lower values near the terminator at $400 \mathrm{~km}$. Local variations of the total magnetic field intensity are also related to the magnitude of the crustal field and are therefore more pronounced in the southern hemisphere.

In this study, we use in situ measurements of the proton energy spectrum made on board the Mars Atmosphere and Volatile Evolution (MAVEN) as an example to calculate the expected Lyman- $\alpha$ auroral line profile and the peak altitude of the expected limb profile. We also model the contribution of the fast $\mathrm{H}$ atom (H-ENAs) precipitation resulting from the interaction of the solar wind protons with the Martian $\mathrm{H}$ corona. In particular, we examine what fraction of the Lyman- $\alpha$ photons produced by collisional processes escape the optically thick hydrogen medium where they are created. We then compare the peak altitude of the modeled limb emission with the recent auroral observations from Mars Express/SPICAM and MAVEN/IUVS. We also discuss the effect of the presence of an induced magnetic field on the Lyman- $\alpha$ line profile and limb intensity distribution. The purpose of this study is not to model or fit any particular observed auroral event, but to investigate the sensitivity of the Lyman- $\alpha$ line profile and peak altitude to the identity of the incident energetic particles (protons or H-ENAs) and the presence of a horizontal induced magnetic field.

\section{Monte Carlo simulations}

Monte Carlo calculations are a necessary first step to determine the directional velocity distribution of the energetic atoms produced in the $2 \mathrm{p} \mathrm{H}$ excited state that relaxes to the $\mathrm{H}$ ground state by emitting the Lyman- $\alpha$ line. The direct simulation Monte Carlo model was adapted to Mars by Shematovich et al. (2011) from the Earth's version used by Gérard et al. (2000) to quantitatively analyze measurements from the SI12 imager of Doppler-shifted Lyman- $\alpha$ auroral emission on board the IMAGE satellite. In brief, the incoming $\mathrm{H}-\mathrm{H}^{+}$beam interacts with the main atmospheric constituents through momentum and energy transfer in elastic and inelastic collisions, ionization of target molecules or atoms, charge transfer and electron capture collisions. Energetic $\mathrm{H}$ atoms or protons produced during the collisions further collide with the atmospheric constituents, and transfer a part of their momentum and kinetic energy to atmospheric particles. The corresponding cross sections and scattering angle distributions for $\mathrm{H} / \mathrm{H}^{+}$interactions have been described by Shematovich et al. (2011). The atmospheric region under study is divided into 49 vertical cells. The cell sizes are chosen in such a way that their size must be equal to or smaller than the particle free path length. The position and the three velocity components for each modeling particle are stored. The modeling particle trajectory is calculated in a 3-D space for each time step and the resulting radial position is preserved for the next step. Therefore, protons launched at given angle relative to the nadir direction can move to different angles. The planetary curvature is also taken into account. As the initial proton (or $\mathrm{H}$ atom) beam interacts with the Martian atmosphere, the $\mathrm{H} / \mathrm{H}^{+}$ ratio reaches an equilibrium controlled by the relative magnitude of the cross sections involved. An essential aspect of this model is the stochastic treatment of the scattering angle distribution following collisions (Shematovich et al., 2011).

The model lower boundary is located at $80 \mathrm{~km}$, well below the homopause. The upper boundary is at $500 \mathrm{~km}$, essentially above the region of the interaction with the Martian thermosphere. The neutral composition and temperature are adopted from Fox and Hać (2009) for a low level of solar activity. The input flux at the top of the model is based on in situ measurements of the incoming protons measured with the SWIA instrument in the planetary sheath region described by Halekas et al. (2015). The effect of an induced magnetic field is simulated by adding a uniform, time independent horizontal B-field, in agreement with the observations by the Mars Global Surveyor (MGS) (Brain et al., 2003) and the Mars Express orbiters (Akalin et al., 2010). In the presence of a magnetic field, the penetration of protons in the thermosphere is also controlled by the value of their gyroradius that is proportional to the square root of their energy and inversely proportional to the B-field intensity. If the gyroradius is smaller than the distance from the top of the model to the peak altitude, the proton has a reduced probability to produce Lyman- $\alpha$ emission (the magnetic barrier). The efficiency of this barrier is energy dependent: those protons with a lower energy have a smaller gyroradius and are therefore more efficiently backscattered than the more energetic population. The induced magnetic field partially acts as a high-pass filter. This effect is stochastically implemented in the Monte Carlo code as in the study by Shematovich et al. (2011).

A second step involves the calculation of the production of $\mathrm{H}$ atoms in the $2 p$ excited state that relaxes to the $\mathrm{H}$ ground state by emitting the Lyman- $\alpha$ line. Three processes produce fast excited hydrogen atoms:

$\begin{array}{lll}\mathrm{H}_{\mathrm{f}}^{+}+\mathrm{M} \rightarrow \mathrm{H}_{\mathrm{f}}+\mathrm{M}^{+} & \text {(1) } & \text { (charge } \\ \mathrm{H}_{\mathrm{f}}+\mathrm{M} \rightarrow \mathrm{H}_{\mathrm{f}}{ }_{\mathrm{f}}+\mathrm{M} & \text { (2) } & \text { (insfer) } \\ & & \text { (inelastic colli- } \\ \mathrm{H}_{\mathrm{f}}+\mathrm{M} \rightarrow \mathrm{H}_{\mathrm{f}}+\mathrm{M}^{+}+\mathrm{e} & \text { (3) } & \text { (ion) } \\ \text { ionization) }\end{array}$

where $\mathrm{M}$ denotes a background neutral constituent, $\mathrm{H}^{+}$energetic protons, $\mathrm{H}_{\mathrm{f}}$ fast hydrogen atoms, and $\mathrm{H}_{\mathrm{f}}$ fast $\mathrm{H}$ atoms in the $2 \mathrm{p}$ excited state. The cross sections for Ly- $\alpha$ excitation for $\mathrm{M}=\mathrm{CO}_{2}, \mathrm{~N}_{2}$ and $\mathrm{O}$ are taken from Haider et al. (2002) using the analytical formula given by Rudd et al. (1985). In the case of $\mathrm{CO}_{2}$, the main target constituent below $\sim 200 \mathrm{~km}$, the cross section for process (1) peaks near $900 \mathrm{eV}$ and for (2) and (3) at $\sim 2 \mathrm{keV}$. We note that the thermal hydrogen atoms in the thermosphere play no role in the production of Lyman- $\alpha$ photons. At each altitude grid point, the velocity distribution functions of the $\mathrm{H}_{\mathrm{f}}$, fast excited atoms is calculated in all directions so that contributions to the formation of the Lyman- $\alpha$ line at Doppler-shifted wavelengths may be accumulated for any given direction of observation. Finally, the observed line profile and the total intensity at the limb results from the summed production of Lyman- $\alpha$ photons in collisional processes (1), (2) and (3) combined with absorption by $\mathrm{CO}_{2}$ along the line of sight below about $100 \mathrm{~km}$.

\section{Results}

We now describe the results of the calculated Lyman- $\alpha$ line profile and simulated limb profile distribution based on the proton flux input measured with the SWIA instrument on board MAVEN. The effect of an 
induced magnetic field and the role of the fraction of H-ENA precipitation at the top of the model are also examined. The proton and $\mathrm{H}$ ENA energy distribution functions (EDF) for the cases illustrated in this study were calculated using the Monte Carlo model. The variation of these energy distribution functions with altitude for different values of the induced magnetic field was illustrated in Bisikalo et al. (2018). For simplicity, we assume that the flux of protons and $\mathrm{H}$ atoms at the top of the model is isotropic. Numerical simulations have shown that this assumption that does not critically affect the line shape nor the vertical distribution of the emission.

\subsection{Lyman- $\alpha$ auroral line profile}

We calculate the emitted Lyman- $\alpha$ line profile by summing the contributions of the excitation processes at each wavelength for different values of the tangent point altitude. The angle between the local vertical direction and the line of sight varies between $90^{\circ}$ at the tangent point and $0^{\circ}$ at infinity. To calculate the resulting line profile and the emerging intensity in any given direction, we integrate all contributions of Lyman- $\alpha$ photons produced in collisional processes (1), (2) and (3) following projection of the velocity vectors along the line of sight. Since collisions between H-ENAs and protons are negligible, we treat separately the cases of proton and H-ENA precipitation through the model upper boundary. The precipitation at $500 \mathrm{~km}$ is composed of both types of particles and their contribution can be combined at each altitude to determine the characteristics of the emergent emission. The value of the $\mathrm{H}^{+} / \mathrm{H}$-ENA flux ratio at the model upper boundary will be discussed in Section 4. In a first step, we neglect absorption by $\mathrm{CO}_{2}$ along the line of sight to isolate the role of the fast $\mathrm{H}$ atom velocity distribution in the formation of the line profile. The role of absorption by $\mathrm{CO}_{2}$ on the emerging intensity will be introduced in the next section when we discuss the characteristics of the simulated limb brightness.

We first model the Lyman- $\alpha$ line profile excited by an initial precipitating flux solely composed of precipitating protons at the top of the model (Case 1). We use the proton flux measured with SWIA in the magnetosheath on 24 March 2015 illustrated in Fig. 1 by Halekas et al. (2015). It was characterized by an energy spectrum with a main peak at $\sim 400 \mathrm{eV}$, a fast drop beyond $2 \mathrm{keV}$ and a total precipitated energy flux of $0.15 \mathrm{~mW} \mathrm{~m}^{-2}$. The corresponding proton and fast hydrogen velocity distributions calculated as a function of altitude were described by Bisikalo et al. (2018). We then use the directional $\mathrm{H}$ (2p) velocity vector field as a function of altitude to determine their projection and integrate all contributions along any simulated line of sight. Fig. 1 shows the line profiles calculated using the measured sheath proton spectrum for a limb observation from a spacecraft at $350 \mathrm{~km}$, the typical altitude of the Mars Express orbiter during several proton auroral signature detections (Ritter et al., 2018). In this and the following two plots, absorption by $\mathrm{CO}_{2}$ has been set to zero in order to isolate the effect of the changing tangent height altitude on the line profile. Both the line profile and the intensity at rest wavelength vary with the altitude of the tangent point. The maximum value is located near $129 \mathrm{~km}$, close to the altitude of maximum energy deposition in the atmosphere. We note that the profiles are symmetric and that the shapes of the far wings are nearly identical at all five tangent altitudes. The full width at half maximum (FWHM) of the line profile is $0.74 \AA$ at $130 \mathrm{~km}$ and the central emission rate is $4.7 \mathrm{kR} \AA^{-1}$. It broadens at higher tangent altitudes as a result of the higher mean energy of the fast $\mathrm{H}$ atoms.

The effect of a moderate induced magnetic field is illustrated in Fig. 2 where the line profiles have been calculated with the same incident proton flux input at the top of the model. In this case, a horizontal field of $15 \mathrm{nT}$ has been added in the stochastic implementation of the steady state Boltzmann equation. The peak intensity at rest wavelength is then predicted at $120 \mathrm{~km}$ with a FWHM of $1.04 \AA$. If the B intensity increases to $20 \mathrm{nT}$, the line width is $1.28 \AA$. It reaches $2.35 \AA$ for $\mathrm{B}=40 \mathrm{nT}$. Comparing Figs. 1 and 2 and Table 1, two main

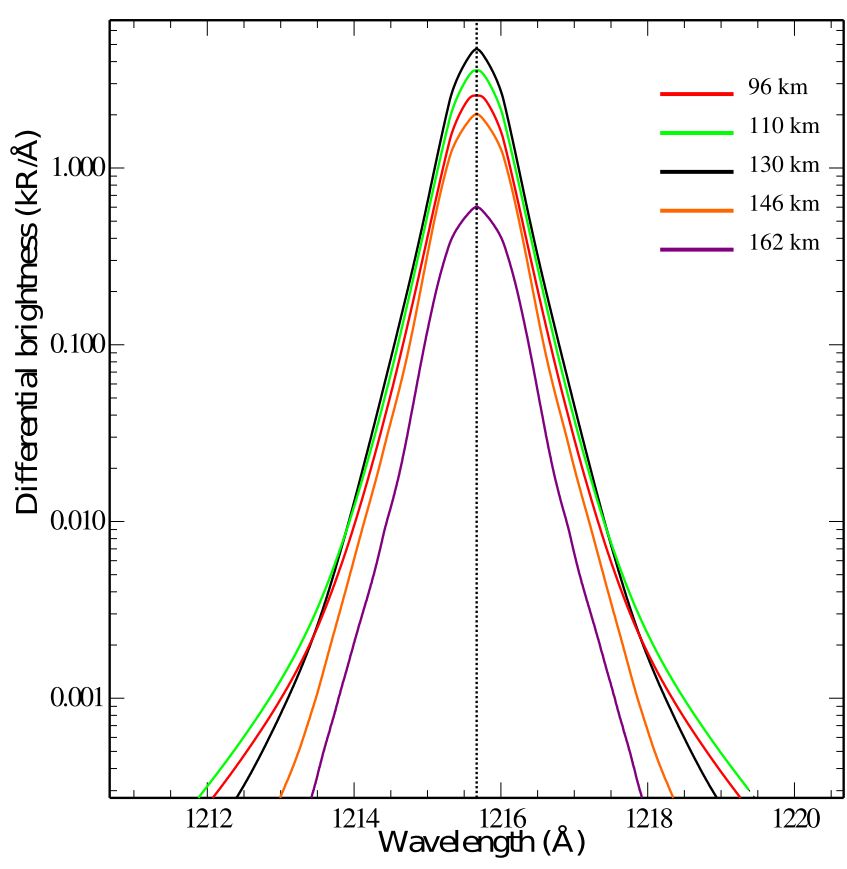

Fig. 1. Auroral Ly- $\alpha$ line profile at the limb from an altitude of $350 \mathrm{~km}$ for energetic proton precipitation calculated for five different values of the tangent altitude of the line of sight. The induced magnetic field intensity is set to zero. The vertical dotted line indicates the rest wavelength $(121.56 \mathrm{~nm})$. The maximum intensity is reached at an altitude of $130 \mathrm{~km}$. Absorption by $\mathrm{CO}_{2}$ is not accounted for in this plot.

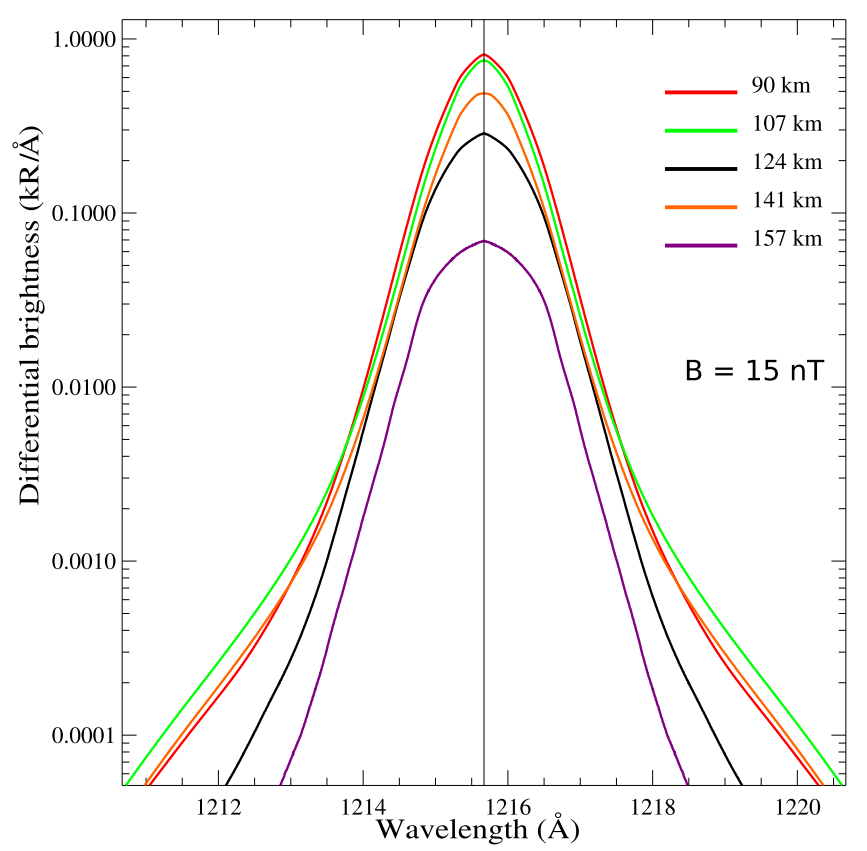

Fig. 2. Same as Fig. 1 in the presence of a $15 \mathrm{nT}$ induced magnetic field. The maximum intensity is reached at a tangent point altitude of $124 \mathrm{~km}$.

differences may be noticed: i) the brightness of the full line profile is significantly reduced in the presence of the induced B-field as expected from the magnetic barrier effect acting on the proton component, and ii) the line width has increased at all altitudes.

Fig. 3 shows the calculated line profile in the case of precipitation of H-ENAs only through the model upper boundary (Case 2), in the absence of a magnetic field. The $\mathrm{H}$ energy spectrum is assumed to be equal to the proton spectrum in the solar wind reported by Halekas et al. (2015) and shown in the bottom right panel of their 
Table 1

Summary of Monte Carlo simulation results (including absorption by $\mathrm{CO}_{2}$ ).

\begin{tabular}{lllll}
\hline $\begin{array}{l}\text { Precipitating } \\
\text { particle }\end{array}$ & $\begin{array}{l}\text { Induced B- } \\
\text { field (nT) }\end{array}$ & $\begin{array}{l}\text { Ly- } \alpha \text { line } \\
\text { width }(\AA)\end{array}$ & $\begin{array}{l}\text { Ly- } \alpha \text { peak } \\
\text { altitude }(\mathrm{km})\end{array}$ & $\begin{array}{l}\text { Ly- } \alpha \text { peak } \\
\text { brightness (kR) }\end{array}$ \\
\hline $\mathrm{p}$ & 0 & 0.74 & 129 & 3.6 \\
$\mathrm{H}-$-ENAs & 0 & 0.94 & 125 & 22.0 \\
$\mathrm{p}$ & 15 & 1.04 & 123 & 0.8 \\
$\mathrm{H}$-ENAs & 15 & 1.13 & 126 & 8.0 \\
$\mathrm{p}$ & 20 & 1.28 & 120 & 0.4 \\
H-ENAs & 20 & 1.29 & 127 & 2.3 \\
$\mathrm{p}$ & 40 & 2.35 & 116 & 0.013 \\
H-ENAs & 40 & 0.91 & 125 & 0.003 \\
\hline
\end{tabular}

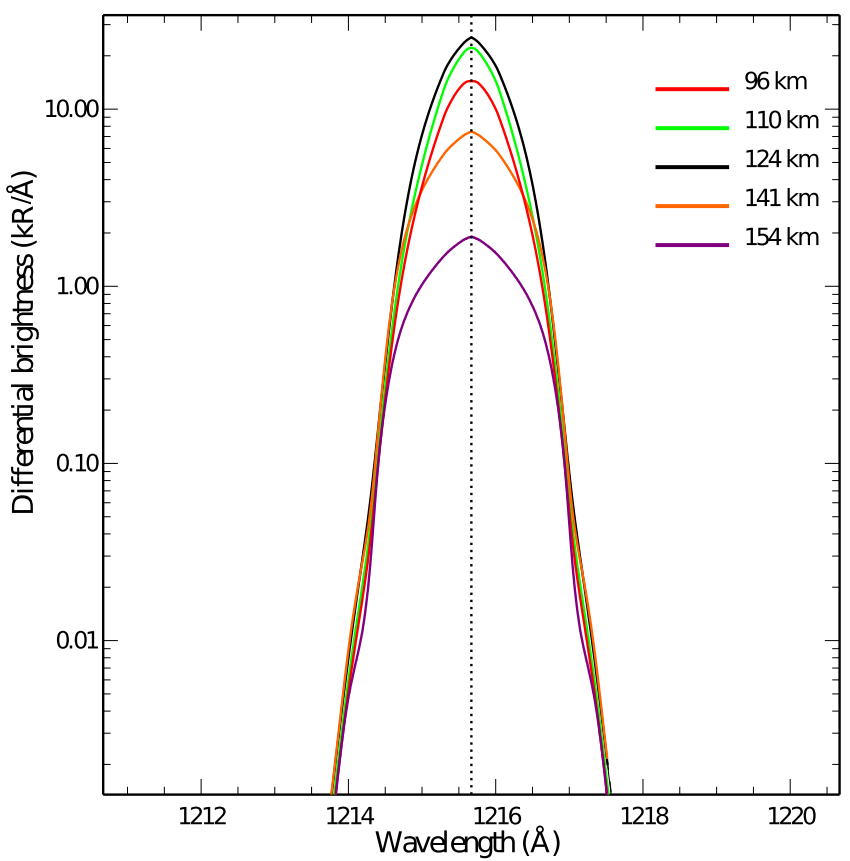

Fig. 3. Same as Fig. 1 for energetic hydrogen atom precipitation in the absence of an induced magnetic field. The maximum intensity is reached at a tangent point altitude of $124 \mathrm{~km}$.

Fig. 1. It is also identical to that used in the recent JGR paper by Bisikalo et al. (2018) plotted in green in their Figs. 4, 5 and 6. The corresponding total downward energy flux is equal to $0.81 \mathrm{~mW} \mathrm{~m}^{-2}$ The line FWHM at the emission peak at $125 \mathrm{~km}$ is then $0.94 \AA$, that is $27 \%$ wider than for proton precipitation. Simulations including the presence of an induced field give a Lyman- $\alpha$ FWHM at the maximum emission of $1.13,1.29$ and $0.91 \AA$ for $\mathrm{B}=15,20$ and $40 \mathrm{nT}$ respectively. Based on these auroral line profiles we now discuss the efficiency of the Lyman- $\alpha$ escape from the optically thick Martian thermosphere.

\subsection{Lyman- $\alpha$ optical thickness}

The fraction of the auroral Lyman- $\alpha$ photons escaping the Martian atmosphere without scattering by thermal hydrogen atoms may be estimated by comparing the auroral line width with the thermal $\mathrm{H}$ atom width. The thermal line profile is Gaussian with a full width at half maximum (FWHM) of $12 \mathrm{~m} \AA$ at $200 \mathrm{~K}$, a typical temperature prevailing in the Martian daytime thermosphere. The self-absorption cross section at the line center is given in $\mathrm{cm}^{2}$ by the expression $\sigma_{0}=5.9 \times 10^{-12}$ / $\mathrm{T}^{1 / 2}$, where $\mathrm{T}$ is the local gas temperature in Kelvin. We adopt the thermal $\mathrm{H}$ vertical density distribution in the thermosphere given by Krasnopolsky (2002) for moderate solar activity. The path of the photons emitted near the line center is modified by multiple scattering close to the rest wavelength, but is decreasingly affected for the Doppler-shifted photons in the line wings. The vertical self-absorption
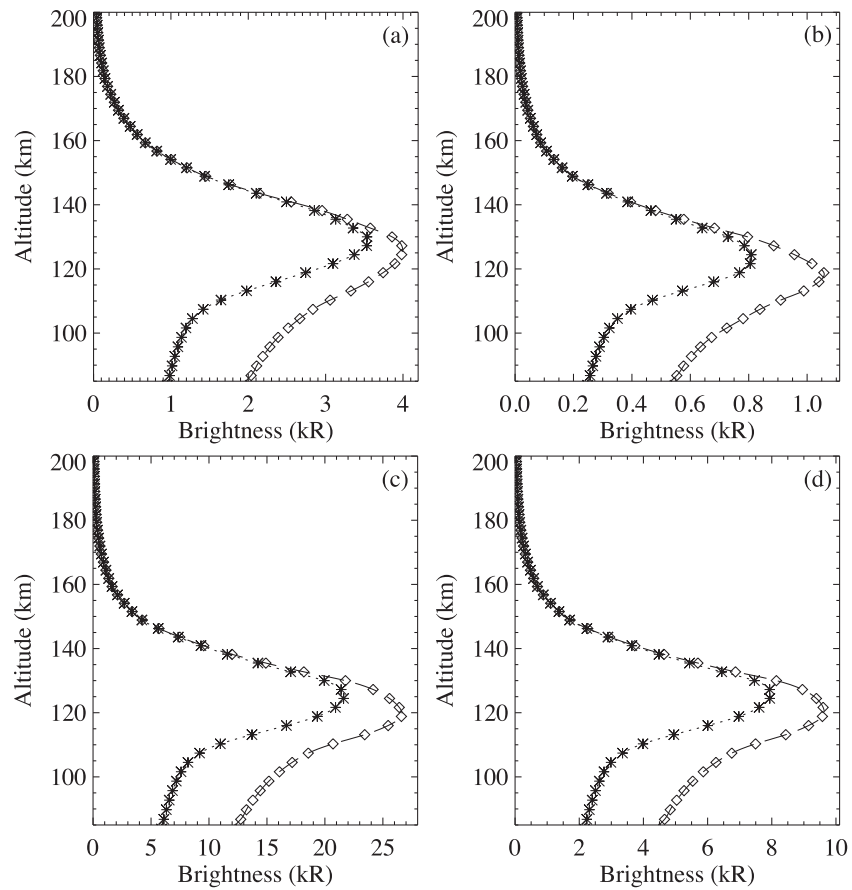

Fig. 4. (a) Calculated distribution of auroral Lyman- $\alpha$ limb intensity for proton precipitation in the absence of an induced magnetic field (see text). Diamonds: without $\mathrm{CO}_{2}$ absorption, stars: including the effect of $\mathrm{CO}_{2}$ absorption along the line of sight for an observer at $350 \mathrm{~km}$; (b) same for a $15 \mathrm{nT}$ induced magnetic field; (c) same as (a) for fast $\mathrm{H}$ atom precipitation; (d) same as (c) for a $15 \mathrm{nT}$ induced magnetic field.

optical depth at rest wavelength is 4.4 at $130 \mathrm{~km}$ and reaches 22 at the limb for a tangent altitude of $130 \mathrm{~km}$, so that the atmosphere is optically thick for those photons having a wavelength near the rest wavelength. However, the Doppler width of the thermal Gaussian $\mathrm{H}$ is about 62 times less than the FWHM of the auroral profile calculated for Case 1. Therefore, the auroral photons emitted a few thermal Doppler widths from line center are largely unaffected by the presence of thermal hydrogen. Comparison with the auroral width previously described shows that the optically thick fraction of the emitted photons is $\sim 3 \%$, in the case of protons precipitation without induced magnetic field and $\sim 1.5 \%$ with a $15 \mathrm{nT}$ field. These values are defined as the fraction of the simulated line-of-sight integrated emission obtained at a tangent altitude of $130 \mathrm{~km}$ found in a wavelength interval delimited by the wavelength at which the vertical optical thickness (above $130 \mathrm{~km}$ ) has fallen to 0.5 . For this estimate, the whole column was assumed to have a temperature equal to the exospheric temperature, so that our numbers may be viewed as upper limits. If the induced magnetic field is stronger, the line width increases continuously and the fraction of escaping protons becomes increasingly larger.

The large fraction of emerging auroral Lyman- $\alpha$ photons explains why the proton aurora has been be detected by spectrographs with medium spectral resolution such as SPICAM and IUVS. MAVEN is also equipped with a high-resolution echelle spectrograph (McClintock et al., 2014; Mayyasi et al., 2017) providing a spectral resolution of $\sim 8$ $\mathrm{m} \AA$ at Lyman- $\alpha$. Future comparisons with line profiles measured with this instrument will help validating (or not) our concepts on the source of the Martian proton aurora provided that auroral events with sufficient brightness may be observed during the MAVEN mission.

\subsection{Simulated intensity distribution at the limb}

Fig. 4 shows simulated Lyman- $\alpha$ intensity (or radiance) distribution at the limb (integrated over the spectral line profile) for four different assumptions on the identity of the precipitating particle and the 
presence or not of a magnetic field. Case 1 (protons alone, no B-field) is illustrated in Fig. 4a, which shows the simulated limb intensity as a function of the tangent altitude, for an observation from an altitude of $350 \mathrm{~km}$. The curves correspond to the limb distribution calculated without and with absorption by $\mathrm{CO}_{2}$ along the line of sight. Similarly to the line profiles discussed in Section 3.1 the energy flux carried by the protons through the upper boundary is equal to $0.15 \mathrm{~mW} \mathrm{~m}^{-2}$. In this case, each contribution along the line of sight is attenuated exponentially according to the Beer-Lambert law. The $\mathrm{CO}_{2}$ absorption cross section at $1215.57 \AA$ is equal to $6.1 \times 10^{-20} \mathrm{~cm}^{2}$ at $195 \mathrm{~K}$ (Yoshino et al., 1996). Consequently, $\tau=1$ corresponds to a $\mathrm{CO}_{2}$ slant density equal to $1.6 \times 10^{19} \mathrm{~cm}^{-2}$ and the emission is totally absorbed below $\sim 80 \mathrm{~km}$. These curves indicate that absorption by $\mathrm{CO}_{2}$ becomes important in the limb viewing geometry below $\sim 130 \mathrm{~km}$. In this case, absorption causes an upward displacement of the brightness peak by about $5 \mathrm{~km}$ to $129 \mathrm{~km}$ and drop from 4 to $3.6 \mathrm{kR}$. The simulated intensity drops by a factor of 2 at $88 \mathrm{~km}$. The case for a horizontal component of the induced magnetic field of $15 \mathrm{nT}$ is shown in panel 4(b). The presence of the field generates maximum limb intensity at $123 \mathrm{~km}$ with a calculated peak brightness of $0.8 \mathrm{kR}$, that is more than 4 times less than without a magnetic field. Simulation for $\mathrm{B}=20 \mathrm{nT}$ yield a peak at $120 \mathrm{~km}$ and a brightness as low as $0.38 \mathrm{kR}$. These results are summarized in Table 1. This decrease of the intensity is further pronounced for a B-field intensity of $40 \mathrm{nT}$. In this case, the gyroradius of a $1 \mathrm{keV}$ proton is $114 \mathrm{~km}$, much smaller than the $457 \mathrm{~km}$ for $10 \mathrm{nT}$ and $228 \mathrm{~km}$ for $20 \mathrm{nT}$. Most protons are then backscattered before interacting with the bulk of the thermosphere, resulting in a very low intensity of less than $30 \mathrm{R}$ Lyman- $\alpha$ with a peak production at the limb located at $107 \mathrm{~km}$ and a peak emission at $116 \mathrm{~km}$ when accounting for $\mathrm{CO}_{2}$ absorption.

As mentioned before, charge exchange reactions of solar wind protons with the exosphere produce H-ENAs that impact the Martian atmosphere. In this process, a solar wind proton captures an electron from an atmospheric atom and produces a fast $\mathrm{H}$ atom and a new cold proton. Case 2 (precipitation of fast H-ENAs only at the top of the model) is illustrated in Fig. $4 \mathrm{c}$ for the $\mathrm{B}=0$ case. It shows a maximum limb intensity of $22 \mathrm{kR}$ at $125 \mathrm{~km}$. As before, this curve was calculated for an initial fast $\mathrm{H}$ atom flux equal to the proton solar wind flux measured by the SWIA instrument, with a total energy flux of $0.81 \mathrm{~mW}$ $\mathrm{m}^{-2}$. A test case considering the same H-ENA precipitation in the presence of a $15 \mathrm{nT}$ B-field was also conducted. The peak brightness then drops to $8 \mathrm{kR}$ at a tangent height of $126 \mathrm{~km}$. For B $=20 \mathrm{nT}$, the peak altitude is nearly unchanged. However, the peak brightness is nearly 10 times less than the $\mathrm{B}=0$ simulation. In the case of a $40 \mathrm{nT}$ field intensity, the calculated peak brightness is at $120 \mathrm{~km}$ if not accounting for absorption by $\mathrm{CO}_{2}$, and $125 \mathrm{~km}$ as a result of strong absorption of the lower part of the emission profile.

Table 1 summarizes the results for the altitudes of the maximum intensity described before. The corresponding Lyman- $\alpha$ intensities are given for simulations including absorption by $\mathrm{CO}_{2}$. Comparison of these results leads to a few conclusions:

i) The width of the Lyman- $\alpha$ auroral line increases with the strength of the horizontal magnetic field. This leads to a growing fraction of the produced photons able to escape the atmosphere and be observed from outside the planet.

ii) The line width of the Lyman- $\alpha$ line, and thus the fraction of escaping protons, is larger for $\mathrm{H}$ atoms than for proton precipitation.

iii) The altitude of the emission peak for proton precipitation decreases when the induced field intensity increases.

iv) The emerging intensity decreases with increasing B-field magnitude for both proton and H-ENA precipitation as a result of the combined effects of preferential backscattering of the lower energy proton component (with smaller gyroradius) and increased absorption by $\mathrm{CO}_{2}$ associated with the altitude drop.

v) In the case of H-ENA precipitation, the altitude of maximum emission remains quite insensitive to the value of the induced magnetic field. The incoming fast $\mathrm{H}$ atoms penetrating into the exosphere remain essentially unaffected by the magnetic field until they reach the denser collisional region where they start efficiently loosing their energy and get ionized, thus becoming sensitive to the intensity of the magnetic field.

\section{Discussion and conclusion}

The altitude of maximum Lyman- $\alpha$ limb intensity observed with SPICAM ranges from 121 to $148 \mathrm{~km}$, with a mean value of $135 \mathrm{~km}$. The emission peak altitudes in the cases from MAVEN-IUVS illustrated by Deighan et al. (2018) are located at 130 and $135 \mathrm{~km}$. The predicted auroral emission peak based on the proton flux measured with SWIA in the magnetosheath is located at $129 \mathrm{~km}$ and $123 \mathrm{~km}$ without and with a $15 \mathrm{nT}$ induced field respectively in close agreement with the observations. In the case of pure fast $\mathrm{H}$ atom precipitation, the Monte Carlo calculation predicts a peak near $125 \mathrm{~km}$. The close agreement with the observed altitude range indicates that a combination of precipitated protons and H-ENAs with an energy spectrum similar to those used as input in this model can produce Lyman- $\alpha$ peaks that are consistent with those observed with SPICAM and IUVS. Measurements of the $\mathrm{H}^{+} / \mathrm{H}-$ ENA flux ratio at the top of the Monte Carlo model have not been discussed so far in the literature. In a recent study, Wang et al. (2018) have modeled the properties of $\mathrm{H}$ atoms precipitation into the atmosphere using a global hybrid plasma model. They find that the value of the $\mathrm{H}^{+} / \mathrm{H}$-ENA ratio at the upper boundary in their model rapidly drops from over 100 beyond $1600 \mathrm{~km}$ to $\sim 1$ near $600 \mathrm{~km}$ (X.D. Wang, private communication).

Halekas et al. (2017) analyzed SWIA measurements to characterize the interaction of the solar wind with the Martian environment. They confirmed the order-of-magnitude seasonal variation of the corona neutral hydrogen content previously deduced from observations of the coronal thermal Lyman- $\alpha$ intensity (Clarke et al., 2014, 2017; Chaffin et al., 2014; Bhattacharyya et al., 2015). We note that the seasonal variation of the $\mathrm{H}$ column density in the exosphere can modify the value of this ratio at the upper boundary of our model. However, it will not affect the interaction of the incident particle beam with the thermosphere since atomic hydrogen plays no significant role in the region of the Monte Carlo simulations described here. Once this ratio and the intensity of the induced magnetic field are known, the characteristics of the Lyman- $\alpha$ limb distribution may be calculated with this model.

The auroral intensities observed so far are quite variable and range from $\sim 0.1 \mathrm{kR}$ up to $\sim 3 \mathrm{kR}$ (Ritter et al., Table 1; Deighan et al., 2018). A detailed intensity and altitude comparison to the observations is not possible at this stage as the proton flux measurements used as an input to the Monte Carlo model, the fraction of H-ENAs in the beam at the model upper boundary, the induced magnetic field and the Lyman- $\alpha$ enhancements have not been measured simultaneously. One may however note that the observed peak values are within the range between $4.3 \mathrm{kR}$ calculated for proton precipitation for $\mathrm{B}=0$ and less than $1 \mathrm{kR}$ for a field intensity larger than $15 \mathrm{nT}$. Similarly, for H-ENA only precipitation, the calculated intensity ranges from $22 \mathrm{kR}$ for $\mathrm{B}=0$ to less than $1 \mathrm{kR}$ for $\mathrm{B}=20 \mathrm{nT}$. If the precipitated $\mathrm{H}$ atom energy flux is divided by $0.81 / 0.15=5.4$ to equal the proton energy flux, for H-ENA only precipitation the calculated intensity ranges from $4.1 \mathrm{kR}$ for $\mathrm{B}=0$ to less than $0.1 \mathrm{kR}$ for $\mathrm{B}=20 \mathrm{nT}$.

As an example, if the downward energy fluxes carried by the protons and the H-ENAs are equal at $500 \mathrm{~km}$ as suggested by the recent model by Wang et al. (2018), our simulations predict a combined Lyman- $\alpha$ limb intensity of $2.3 \mathrm{kR}$ for $\mathrm{B}=15 \mathrm{nT}$ and $0.8 \mathrm{kR}$ for a $20 \mathrm{nT}$ induced field for the flux conditions used in these simulations. These values are in line with the observed peak intensity of the strongest detected proton auroral events published so far. The brightness is expected to drop to considerably smaller values as the horizontal induced 
field intensity increases. The calculated altitude of the maximum limb intensity is 125 and $124 \mathrm{~km}$ respectively.

We note that the results presented here are for a purely horizontal orientation of the magnetic field. Any deviation from horizontality, which are highly likely to occur, will decrease the efficiency of the magnetic barrier and actually guide protons deeper into the atmosphere. The actual particle flux interacting with the atmosphere is a mixture of protons and H-ENAs whose value is likely to vary with the hydrogen content of the exosphere, solar activity and related solar wind perturbations. If $\mathrm{H}$ atoms make an increasing fraction of the incoming flux, the Lyman- $\alpha$ intensity is expected to increase in comparison with a pure proton flux. Our simulations also suggest that the total magnetic field intensity significantly controls the efficiency of the $\mathrm{H}^{+}-\mathrm{H}$ beam in producing Lyman- $\alpha$ proton aurora. The induced field has been measured to reach values as large as $40 \mathrm{nT}$ at $400 \mathrm{~km}$ near the subsolar point (Akalin et al., 2010). In this case, one might expect that these regions should be associated with low or absent auroral Lyman- $\alpha$ emission. Future observations with the IUVS spectrograph on board MAVEN should provide useful information on the statistical geographic, local time and altitude distribution of the dayside proton aurora.

An interesting result is that, although the value of the absorption cross section at Lyman- $\alpha$ wavelength is quite low, absorption by $\mathrm{CO}_{2}$ plays a role in the limb intensity distribution below about $130 \mathrm{~km}$. The wide line profile of the calculated Lyman- $\alpha$ auroral emission at the limb is almost exactly symmetric with respect to the rest wavelength as a consequence of the symmetry of the limb observing geometry. A very weak asymmetry and peak intensity wavelength shift could be caused by the differential absorption by $\mathrm{CO}_{2}$ of the section of the line of sight located in front of or beyond the tangent point in the case of observations from a spacecraft close to the emitting layer.

The sources of Lyman- $\alpha$ photons considered in this study are collisions described by relations (1) to (3) leading to the production of $\mathrm{H}$ (2p) excited atoms. Another possible contribution to the production of auroral Lyman- $\alpha$ photons on the dayside is resonance scattering of solar radiation by hot $\mathrm{H}$ atoms. Collisions of the $\mathrm{H}^{+}-\mathrm{H}$ beam likely generate an enhanced density of non-thermal atoms in the Martian upper thermosphere. These hot atoms are able to scatter solar photons far from the thermal core and may lead to an increased Lyman- $\alpha$ brightness in regions submitted to resonance scattering. However, this contribution does not appear dominant since the maximum hot $\mathrm{H}$ atom density would be located at a higher altitude than the observed emission peak and would likely not exhibit a pronounced maximum as reported in the observations. Future work based on a much larger sample of proton aurora detections concurrent with measurements of proton energy spectra will be useful to clarify issues such as the dependence of frequency and intensity on local time, solar zenith angle and solar activity.

\section{Acknowledgements}

B.H. is supported by the Fonds National de la Recherche Scientifique (FNRS) D.B. and V.S. were supported by the Russian Science Foundation (Project 18-12-00447). This research was partly funded by the SCOOP/BRAIN program of the Belgian Federal Science Policy Office (BELSPO). We thank Dr. X.D. Wang et al. for providing model results for the altitude dependence of the H-ENAs fraction.

\section{References}

Akalin, F., Morgan, D.D., Gurnett, D.A., Kirchner, D.L., Brain, D.A., Modolo, R., Espley, J.R., 2010. Dayside induced magnetic field in the ionosphere of Mars. Icarus 206 (1), 104-111.

Bhattacharyya, D., Clarke, J.T., Bertaux, J.L., Chaufray, J.Y., Mayyasi, M., 2015. A strong seasonal dependence in the Martian hydrogen exosphere. Geophys. Res. Lett. 42 (20),
8678-8685.

Bertaux, J.L., Leblanc, F., Witasse, O., Quemerais, E., Lilensten, J., Stern, S.A., Korablev, O., 2005. Discovery of an aurora on Mars. Nature 435 (7043), 790-794.

Bisikalo, D.V., Shematovich, V.I., Gérard, J.C., Hubert, B., 2017. Influence of the crustal magnetic field on the Mars aurora electron flux and UV brightness. Icarus 282, 127-135.

Bisikalo, D.V., Shematovich, V.I., Gérard, J.C., Hubert, B., 2018. Monte Carlo simulations of the interaction of fast proton and hydrogen atoms with the Martian atmosphere and comparison with in situ measurements. J. Geophys. Res. https://doi.org/10. 1029/2018JA025400.

Brain, D.A., Bagenal, F., Acuña, M.H., Connerney, J.E.P., 2003. Martian magnetic morphology: contributions from the solar wind and crust. J. Geophys. Res. 108 (A12)

Chaffin, M.S., Chaufray, J.Y., Stewart, I., Montmessin, F., Schneider, N.M., Bertaux, J.L., 2014. Unexpected variability of Martian hydrogen escape. Geophys. Res. Lett. 41 (2), 314-320.

Clarke, J.T., Bertaux, J.L., Chaufray, J.Y., Gladstone, G.R., Quémerais, E., Wilson, J.K., Bhattacharyya, D., 2014. A rapid decrease of the hydrogen corona of Mars. Geophys. Res. Lett. 41 (22), 8013-8020.

Clarke, J.T., et al., 2017. Variability of D and H in the Martian upper atmosphere observed with the MAVEN IUVS echelle channel. J. Geophys. Res. Space Phys. 122, 2336-2344. https://doi.org/10.1002/2016JA023479.

Fox, J.L., Hać, A.B., 2009. Photochemical escape of oxygen from Mars: A comparison of the exobase approximation to a Monte Carlo method. Icarus 204, 527-544.

Deighan, J., Jain, S.K., Chaffin, M.S., Fang, X., Halekas, J.S., Clarke, J.T., Stevens, M.H., 2018. Discovery of a proton aurora at Mars. Nature Astro. 1.

Gérard, J.C., Hubert, B., Bisikalo, D.V., Shematovich, V.I., 2000. A model of the Lyman- $\alpha$ line profile in the proton aurora. J. Geophys. Res. 105 (A7), 15795-15805. https:// doi.org/10.1029/1999JA002002.

Gérard, J.C., Soret, L., Libert, L., Lundin, R., Stiepen, A., Radioti, A., Bertaux, J.L., 2015. Concurrent observations of ultraviolet aurora and energetic electron precipitation with Mars Express. J. Geophys. Res. 120, 6749-6765.

Gérard, J.C., Soret, L., Shematovich, V.I., Bisikalo, D.V., Bougher, S.W., 2017. The Mars diffuse aurora: a model of ultraviolet and visible emissions. Icarus 288, 284-294.

Haider, S.A., Seth, S.P., Kallio, E., Oyama, K.I., 2002. Solar EUV and electron-protonhydrogen atom-produced ionosphere on Mars: comparative studies of particle fluxes and ion production rates due to different processes. Icarus 159 (1), 18-30.

Halekas, J.S., Lillis, R.J., Mitchell, D.L., Cravens, T.E., Mazelle, C., Connerney, J.E.P. Ruhunusiri, S., 2015. MAVEN observations of solar wind hydrogen deposition in the atmosphere of Mars. Geophys. Res. Lett. 42 (21), 8901-8909.

Halekas, J.S., et al., 2017. Structure, dynamics, and seasonal variability of the Mars-solar wind interaction: MAVEN solar wind ion analyzer in-flight performance and science results. J. Geophys. Res. Space Physics 122, 547-578. https://doi.org/10.1002/ 2016JA023167.

Kallio, E., Luhmann, J.G., Barabash, S., 1997. Charge exchange near Mars: solar wind absorption and energetic neutral atom production. J. Geophys. Res. 102 (22). https:// doi.org/10.1029/97JA01662. 183-22,197.

Kallio, E., Barabash, S., 2001. Atmospheric effects of precipitating energetic hydrogen atoms on the Martian atmosphere. J. Geophys. Res. 106, 165-177. https://doi.org/ 10.1029/2000JA002003.

Krasnopolsky, V.A., 2002. Mars' upper atmosphere and ionosphere at low, medium, and high solar activities: Implications for evolution of water. J. Geophys. Res. 107 (E12).

Leblanc, F., Witasse, O., Winningham, J., Brain, D., Lilensten, J., Blelly, P.-L., Frahm, R.A., Halekas, J.S., Bertaux, J.L., 2006. Origins of the Martian aurora observed by spectroscopy for investigation of characteristics of the atmosphere of mars (SPICAM) on board Mars express. J. Geophys. Res. 111, A09313. https://doi.org/10.1029/ 2006JA011763.

Mayyasi, M., et al., 2017. IUVS echelle-mode observations of interplanetary hydrogen: standard for calibration and reference for cavity variations between Earth and Mars during MAVEN cruise. J. Geophys. Res. 122, 2089-2105. https://doi.org/10.1002/ 2016JA023466.

McClintock, W., et al., 2014. The imaging ultraviolet spectrograph (IUVS) for the MAVEN mission. Space Sci. Rev, https://doi.org/10.1007/s11214-014-0098-7.

Ritter, B., Gérard, J.-C., Hubert, B., Rodriguez, L., Montmessin, F., 2018. Observations of the proton aurora on Mars with SPICAM on board Mars express. Geophys. Res. Lett. 44. https://doi.org/10.1002/2017GL076235.

Rudd, M.E., Kim, Y.K., Madison, D.H., Gallagher, J.W., 1985. Electron production in proton collisions: total cross sections. Rev. Mod. Phys. 57 (4), 965.

Schneider, N.M., Deighan, J.I., Jain, S.K., Stiepen, A., Stewart, A.I.F., Larson, D., Evans, J.S., 2015. Discovery of diffuse aurora on Mars. Science 350 (6261), aad0313.

Shematovich, V.I., Bisikalo, D.V., Dieval, C., Barabash, S., Stenberg, G., Nilsson, H., Gérard, J.C., 2011. Proton and hydrogen atom transport in the Martian upper atmosphere with an induced magnetic field. J. Geophys. Res. 116 (A11).

Soret, L., Gérard, J.C., Libert, L., Shematovich, V.I., Bisikalo, D.V., Stiepen, A., Bertaux, J.L., 2016. SPICAM observations and modeling of Mars aurorae. Icarus 264, 398-406.

Wang, X.-D., Alho, M., Jarvinen, R., Kallio, E., Barabash, S., 2018. Precipitation of hydrogen energetic neutral atoms at the upper atmosphere of Mars. J. Geophys. Res. 116 (A11) in press.

Yoshino, K., Esmond, J.R., Sun, Y., Parkinson, W.H., Ito, K., Matsui, T., 1996. Absorption cross section measurements of carbon dioxide in the wavelength region $118.7-175.5 \mathrm{~nm}$ and the temperature dependence. J. Quant. Spectrosc. Radiat. Transfer 55 (1), 53-60. 